\title{
What is the future of CCR5 antagonists in rheumatoid arthritis?
}

\author{
Tsutomu Takeuchi* and Hideto Kameda \\ See related research by Fleishaker et al., http://arthritis-research.com/content/14/1/R11
}

\begin{abstract}
Fleishaker and colleagues reported on a doubleblind placebo controlled clinical trial of a C-C chemokine-receptor type 5 (CCR5) antagonist, maraviroc, in rheumatoid arthritis (RA) patients with inadequate response to methotrexate, showing that it was ineffective. Two additional CCR5 antagonists, SCH351125 and AZD5672, also failed to demonstrate clinical efficacy. In addition, CCR5-blocking antibodies could not inhibit synovial fluid-induced monocyte chemotaxis. Thus, CCR5 appears not to be a desirable target in RA treatment. Given the multiple functions of CCR5, redundancies in the chemokine system, and patient selection in the trial, we overview the recent understanding for chemokine receptor blockade in the treatment of RA
\end{abstract}

Rheumatoid arthritis (RA) is an autoimmune disease characterized by both the presence of autoantibodies (either rheumatoid factor or those against citrullinated protein/peptide) and the chronic infiltration of leukocytes into synovial tissue and fluid. The latter is thought to be driven by interactions between chemokines and their G-protein-coupled receptors. Chemokines are known to play important roles in angiogenesis and lymphoid organization, and their expression patterns have been used as markers to identify a subset of lymphocytes and monocytes. As such, chemokines and their receptors have been deemed reasonable targets for the development of new RA treatments.

In a recent article in Arthritis Research $\mathcal{E}$ Therapy, Fleishaker and colleagues [1] reported on the results of a clinical trial of a chemokine receptor antagonist in the treatment of patients with RA. Chemokines are classified

*Correspondence: tsutake@z5.keio.jp

Division of Rheumatology, Department of Internal Medicine, School of Medicine, Keio University, 35 Shinanomachi, Shinjyuku, Toky 160-8582, Japan into $\mathrm{CXC}, \mathrm{CC}, \mathrm{C}$, and $\mathrm{CX} 3 \mathrm{C}$ supergene families according to the number and spacing of conserved cysteines. C-C chemokine-receptor type 5 (CCR5) is abundantly expressed in the RA synovium and T helper-cell type 1 inflammatory infiltrates, and is bound by macrophage inflammatory protein (MIP)-1 $\alpha$ (CCL3), MIP-1 $\beta$ (CCL4), and RANTES (regulated upon activation, normal $\mathrm{T}$ cell expressed, and secreted; CCL5) [2]. A CCR5-deficient mouse model showed reduced bacterial clearance and was protected against endotoxin-induced systemic inflammation and other enhanced immune reactions [3]. Further, although still controversial, a single nucleotide polymorphism resulting in the production of a nonfunctional receptor (CCR5- $\Delta 32)$ protected against $R A$.

These findings have spurred the development of several CCR5 inhibitors. However, in a randomized, double-blind, placebo-controlled clinical trial, Fleishaker and colleagues [1] reported that a CCR5 antagonist (maraviroc), approved for use in HIV patients because CCR5 is the major coreceptor for HIV-1 entry into cells, was ineffective in treating patients with RA who had shown inadequate responses to methotrexate (MTX). Given that their study found no significant clinical efficacy as evaluated based on American College of Rheumatology responder rates or changes from baseline in Disease Activity Score 28-4 C-reactive Protein (DAS28-4 (CRP)), the study was terminated [1]. Similarly, two additional CCR5 antagonists, SCH351125 and AZD5672, respectively tested on RA [4] and MTX-refractory RA patients [5], also failed to demonstrate clinical efficacy. Moreover, neither CCR2nor CCR5-blocking antibodies were able to inhibit synovial fluid-induced monocyte chemotaxis [6]. Therefore, CCR 5 appears not to be a desirable target in RA treatment.

The above-described failures in using CCR5 inhibitors to treat RA may be explained by the multiple functions of CCR5 and redundancies in the chemokine system. However, although the expression of chemokines and their receptors has been believed to be redundant for decades, evidence shows that this may not be the case [7]. Instead, like most developmental processes, a strict temporal and spatial control of their expression could be 
critical in RA pathogenesis. A more fundamental understanding of the pathogenesis and pathophysiology of each RA patient may be needed in order to achieve precise control of the disease through manipulation of the chemokine system. Thus, whether or not drugs are administered to the right patients, at the right time, and with a sufficient dosing regimen in clinical trials is critical.

As for the dosing regimen, that based on conventional pharmacokinetics/pharmacodynamics methods may not have been sufficient to block targeting receptors more than $95 \%$ of the time. A recent review underscored the importance of maintaining dose levels sufficiently above coverage levels for serum A10-receptors [7]. The maximum dosing may be partly limited by non-specific toxicity, such as liver dysfunction, of low molecular weight chemicals and the recycling of CCR5 molecules via the trans-Golgi network [8]. Although the clinical trials targeting inflammatory cytokines such as tumor necrosis factor or interleukin-6 have been consistently positive, while those targeting chemokines have seldom been positive, this may be partly attributable to the differences between biological agents and low molecular weight chemicals, in addition to those between inflammatory cytokines and chemokines. Even with biological agents against tumor necrosis factor, we recently demonstrated via a sub-analysis of the RISING study (a prospective, randomized, double-blind study to compare the efficacy and safety of $10 \mathrm{mg} / \mathrm{kg}$ infliximab with those of $3 \mathrm{mg} / \mathrm{kg}$ infliximab treatment in MTX-refractory RA patients) that the dose of infliximab required to sufficiently neutralize tumor necrosis factor dramatically differs among RA patients [9]. Therefore, even when CCR5 inhibitors may be potentially effective for a small fraction of RA patients, the appropriate dosing regimen of CCR5 inhibitors to achieve sustained receptor occupancy above a sufficient level might be crucial in the successful treatment of RA, but not of HIV infection.

While findings regarding RA treatment with CCR5 inhibitors have thus far proven negative, these results still represent an important milestone in the development of target therapies for RA and other systemic autoimmune and inflammatory diseases.

\section{Abbreviations}

CCR5, C-C chemokine-receptor type 5; MIP, macrophage inflammatory protein; MTX, methotrexate; RA, rheumatoid arthritis,

\section{Competing interests}

The authors declare that they have no competing interests.

\section{Published: 30 March 2012}

\section{References}

1. Fleishaker DL, Meijide JAG, Petrov A, Kohen MD, Wang X, Menon S, Stock TC, Mebus CA, Goodrich JM, Mayer HB, Zeiher BG: Maraviroc, a chemokine receptor-5 antagonist, fails to demonstrate efficacy in the treatment of patients with rheumatoid arthritis in a randomized, double-blind placebo-controlled trial. Arthritis Res Ther 2012, 14:R11.

2. Szekanecz Z, Koch AE, Tak PP: Chemokine and chemokine receptor blockade in arthritis, a prototype of immune-mediated inflammatory diseases. Ned J Med 2011, 69:356-366.

3. Zhou Y, Kurihara T, Ryseck R-P, Yang Y, Ryan C, Loy J, Warr G, Bravo R: Impaired macrophage function and enhanced $T$ cell-dependent immune responses in mice lacking CCR5, the mouse homologue of the major HIV-1 coreceptor. J Immunol 1998, 160:4018-4025.

4. van Kuijk AWR, Vergunst CE, Gerlag DM, Bresnihan B, Gomez-Reino JJ, Rouzier $R$, Verschueren PC, van de Leij C, Maas M, Kraan MC, Tak PP: CCR5 blockade in rheumatoid arthritis: a randomized, double-blind, placebo-controlled clinical trial. Ann Rheum Dis 2010, 69:2013-2016.

5. Gerlag DM, Hollis S, Layton M, Vencovsky J, Szekanecz Z, Braddock M, Tak PP, on behalf of the ESCAPE Study Group: Preclinical and clinical investigation of a CCR5 antagonist, AZD5672, in patients with rheumatoid arthritis receiving methotrexate. Arthritis Rheum 2010, 62:3154-3160.

6. Lebre MC, Vergunst CE, Choi IYK, Aarrass S, Oliveira ASF, Wyant T, Horuk R, Reedquist KA, Tak PP: Why CCR2 and CCR5 blockade failed and why CCR1 blockade might still be effective in the treatment of rheumatoid arthritis. Plos One 2011, 6:e21772.

7. Schall TJ, Proudfoot AEl: Overcoming hurdles in developing successful drugs targeting chemokine receptors. Nat Rev Immunol 2011, 11:355-363.

8. Escola J-M, Kuenzi G, Gaertner H, Foti M, Hartley O: CC chemokine receptor 5 (CCR5) desensitization. Cycling receptors accumulate in the trans-Golgi network. J Biol Chem 2010, 285:41772-41780.

9. Takeuchi T, Miyasaka N, Tatsuki Y, Yano T, Yoshinari T, Abe T, Koike T: Baseline tumour necrosis factor alpha levels predict the necessity for dose escalation of infliximab therapy in patients with rheumatoid arthritis. Ann Rheum Dis 2011, 70:1208-1215.

doi:10.1186/ar3775

Cite this article as: Takeuchi T, Kameda $\mathrm{H}$ : What is the future of CCR5 antagonists in rheumatoid arthritis? Arthritis Research \& Therapy 2012, 14:1 14. 